\title{
The Effect of Using Frankincense (Boswellia sacra) Chewing Gum on the Microbial Contents of Buccal/Oral Cavity, Taif, KSA
}

\author{
Dr. Sherifa Mostafa M. Sabra; Luluah Mohammed R. Al-Masoudi \\ (Science College, Taif University, KSA)
}

\begin{abstract}
Frankincense (Boswellia sacra / Oleo-gum-resin), the main effective contents were alcohol-soluble resins is $78 \%$, water-soluble gum $23 \%$ and volatile essential oil $7 \%$ respectively. The contents have antimicrobial effects, on moth flora. The target was to detect the anti-microbial effects on microbial contents of buccal/oral cavity which are the sources of infection. Selection was of 5 female persons aged (25-35yrs.), for the experiment under study as the usage of Frankincense chewing gum for 5hrs. continually. As well as they was tested for microbial contents of buccal/oral cavity through microbial identification of saliva. The main CFUs/ml saliva were 13.000, 8.200, 6.100, 3.900, 2.200, and 740 at Zero, 1, 2, 3, 4, and 5hrs. The differences of CFUs/ml saliva were 4.800, 2.100, 2.200, 1.700, and 1.460 between (Zero $h r .-1 h r.),(1 h r .-2 h r),.(2 h r .-3 h r),.(3 h r .-4 h r$.$) ,$ and (4hr.-5hr.) respectively. CFUs $/ \mathrm{ml}$ saliva were $1.3 \times 10^{4}, 8.2 \times 10^{3}, 6.1 \times 10^{3}, 3.9 \times 10^{3}, 2.2 \times 10^{3}$, and $7.4 \times 10^{2}$ at Zero, 1, 2, 3, 4, 5hr. respectively. Frankincense chewing gum is a safe and low-cost herbal product, applied to improve buccal/oral cavity hygiene by anti-microbial effects which decrease the sources of microbial infection in buccal/oral cavity and support mouth hygiene for all ages. It has been indicated to have wide pharmacological and industrial potentials as well.
\end{abstract}

Key words: Frankincense, Boswellia sacra, Oleo-gum-resin , Buccal, Oral, Microbial, CFUs/ml

\section{Introduction:}

Frankincense (Boswellia sacra) is a tree indigenous to Dhofar region and is one of the most famous plant of Sultanate of Oman. It is also known as Luban, Bakhor or Kendar in Arabic region ${ }^{[1]}$. Its composed of about 5-9\% essential oil, 65-85\% alcohol-soluble resins, and the remaining water soluble gums $30-40 \%{ }^{[2]}$. Oil portion contains $62.1 \%$ ester, $15.4 \%$ alcohol, $9.9 \%$ mono-terpene hydrocarbons and $7.1 \%$ di-terpenes, contain $\alpha-$ pinene, $\alpha$-thujene, $\beta$-pinene, camphene, myrcene, o-methylanisole, $\alpha$-terpinene, methoxytoluene, hexyl acetate, limonene, 1, 8-cineole, n-octanol, linalool, octyl acetate, bornyl acetate, cembrene A, incensole, incensole acetate, sabinene, o-cymene, $\rho$-cymene, 1,8-cineole, cis- $\beta$-ocimene, trans- $\beta$-ocimene, $\Upsilon$-terpinene, 1 -octanol, terpinolene, linalool, 1-decanol, terpinen-4-ol, $\alpha$-terpineol, 1-octyl acetate, citronellyl acetate, neryl acetate, geranyl acetate, hexyl hexanoate, 1-decyl acetate, hexyl octanoate, $\alpha$-campholenic aldehyde, caproic acid, oenanthic acid, caprylic acid, pelargonic acid, lauric acid, and verbenone ${ }^{[3]}$. It's the most important multipurpose tree species in Central and Eastern Africa. possess multiple economic and ecological benefits in Africa. It is found in Ethiopia, Nigeria, Cameroon, Central African Republic, Chad, Sudan, Uganda and Eritrea ${ }^{[4]}$. It produced by several species of Boswellia. "Fran-kincense or Pure- incense" was known to the most of the ancient civilizations who used it in rituals and prayers to the gods. It is used in the traditional medicine in Oman, India and African countries for the treatment of variety of diseases. The oldest written evidence which mentions Frankincense as a drug is papyrus Ebres ${ }^{[5]}$. Essential oil was dominated by $\alpha$-thujene $10 \%$ and pcymene $4-3 \%{ }^{[6]}$. Boswellic acids have an anti-inflammatory action ${ }^{[7]}$, active constituents for many of its medicinal uses ${ }^{[8]}$. Nonsteroidal anti-inflammatory drugs (NSAIDS) used for inflammatory conditions, among the constituents of boswellic acid, $\beta$-boswellic acids (the major constituents of boswellic acids) was shown to have antiinflammatory and anti-arthritic pain activity, which was found to be due to their ability to inhibit 5-lipoxygenase activity $^{[9]}$. Frankincense consists of essential oils, gum, and terpenoids. The boswellic acids in the terpenoid portion are the active constituents in Boswellia ${ }^{[10]}$. The fresh gum obtained from the tree is hot dry with a pleasant flavor and slightly bitter in taste. When burned produces a brilliant flame and produces a pleasant aroma. It was widely used by ancient Egyptians, Greeks and Romans as prized incense, fumigant as well as a multipurpose aromatic. In the past it was used for trading across Asia and Europe. It was the most powerful fuel for the Arab economy as it was once more voluble than gold. The essential oil contains E- $\beta$-ocimene and limonene as the major mono-terpenes $97.3 \%$ however, E- caryophyllene is the main constituent of $2.7 \%$ sesquiterpenes, resin contains a mixture of four penta-cyclic triterpene acids: $\beta$-boswellic acid (the most abundant), 3-O-acetyl $\beta$ )ABA), 11-keto- $\beta$-boswellic acid, and 3-O-acetyl-11-keto- $\beta$-boswellic acid (AKBA), collectively called boswellic acids ${ }^{[11]}$. Boswellic acids are further known to contain $\beta$-boswellic acid, acetyl- $\alpha-$ boswellica acid, and acetyl- $\beta$-boswellic acid, and associated 3-acetyl-11-hrdroxy- $\beta$-boswellic acid and 11-keto$\beta$-boswellic acid acetate chemicals ${ }^{[12]}$. AKBA components of the boswellic acids are also identified to be among the biologically active and most potent constituents ${ }^{[13]}$. Boswellic acid and their derivatives were investigated 
for anti-inflammatory. Essential oil showed anti-bacterial, anti-fungal and immuno-stimulating activity ${ }^{[14]}$. Mono-terpenoids seem to be the dominant class of compounds found in the oils. The main compounds essential oil were $\alpha$-pinene $45.7 \%, \alpha$-terpinene $11.5 \%$ and trans-sabinene hydrate ${ }^{[15]}$. Resin oils showed similar chemical profiles, with iso-incensole and iso-incensole acetate as the main di-terpenic components. Both noctanol and n-octyl acetate, along with the diterpenic components incensole and incensole acetate, were the characteristic compounds of B. papyrifera oleogum resin oil. The anti-microbial activities of the essential oils were individually evaluated against fungi, Gram-positive and Gram-negative bacteria strains. Resins destroy microbial cell wall and stop protein synthesis in Strept. salivaris, Staph. aureus, and Bacillus megaterium ${ }^{[16]}$. Pharmacological activities of Frankincense, as crude extracts, the distilled essential oil and isolated compounds reported to exhibit in-vitro anti-bacterial, anti-fungal and immuno-modulatory activity. Volatile oils make sensitization of microbial cell contents and have anti-inflammatory effect on Staph. aureus, Staph. epidermidis, Staph. hominis, Bacillus cereus, E. coli, Proteus vulgaris and Candida albicans ${ }^{[17]}$. Frankincense application can lead to remarkable decrease in inflammatory indices in comparison to drug therapy. It's a safe and low cost herbal medicine, may be feasibly applied to improve inflammation based disease of gingival as an adjunct to the conventional mechanical therapy ${ }^{[18]}$. The daily and ritual use of Boswellia is characteristic cultural of the horn of Africa which transcends the ethical and religious memberships. The essential oil and methanol extract of Boswellia showed activity against bacterial spp. than against yeast. Water soluble gum has sticky character which stop the reactions between substances ${ }^{[19]}$. In general, most of the respondents were aware of beneficial therapeutic uses of luban, but 3/4 of the participants hardly had an idea that incense is considered as potential chemo preventive agent in modern medicine. Similarly, (38-46\%) of the participants correctly believed that luban is having anti-inflammatory actions, a fact substantiated by scientific evidences and its commercial availability in the pharmaceutical market. More than $1 / 2$ of the study population suggested that luban could be used in cosmetic industry because of its aroma and fragrance. Frankincense was once and still considered to be a national treasure ${ }^{[20]}$. The human mouth is an ideal environment of the existence and growth of micro-organisms. It provides a source of water and nutrients, as well as a moderate temperature. Resident bacteria of the mouth adhere to the teeth and gums to resist mechanical flushing from the mouth to stomach where they are destroyed by hydrochloric acid ${ }^{[21]}$. Anaerobic bacteria in the oral cavity include: Actinomyces, Bacteriodes, Bifidobacterium, Eubacterium, Fusobacterium, Lactobacillus ${ }^{[22]}$. The role of oral micro-organisms in the two major dental diseases: dental caries and periodontal disease. The mouth harbors a diverse, abundant and complex microbial community. This highly diverse micro flora inhabits the various surfaces of the normal mouth. Bacteria accumulate on both the hard and soft oral tissues in biofilms. Bacterial adhesion is particularly important for oral bacteria. Oral bacteria have evolved mechanisms to sense their environment and evade or modify the host. Bacteria occupy the ecological niche provided by both the tooth surface and gingival epithelium. However, a highly efficient innate host defense system constantly monitors the bacterial colonization and prevents bacterial invasion of local tissues. A dynamic equilibrium exists between dental plaque bacteria and the innate host defense system. Oral bacteria include Strept., lactobacilli, staph., Corynebacterium, and various anaerobes in particular Bacteriodes. The oral cavity of the new-born baby does not contain bacteria but rapidly becomes colonized with bacteria such as Strept. salivaris. With the appearance of the teeth during the first year colonization by Strept. mutans and Strept. sanguinis occurs as these organisms colonize the dental surface and gingiva. Other strains of Strept. adhere strongly to the gums and cheeks but not to the teeth. The gingival crevice area (supporting structures of the teeth) provides a habitat for a variety of anaerobic species. Bacteriodes and Spirochetes colonize the mouth around puberty ${ }^{[23]}$. Spirochetes and Fusiform bacilli live as normal flora in the mouth, but in the case of bleeding in the oral cavity, the bacteria can cause infection and diseases to oral cavity. Acute necrotizing ulcerative gingivitis (ANUG), Vincent angina with a membrane covering the throat area. Gram-negative anaerobic Cocci. It is thought that this species thrives in the acidic environment of caries and is thought to slow the development of dental caries. It converts the acidic products of other spp. to less acidic products. Aggregatibacter Actinomycete mcomitans is considered an oral pathogen due to its virulence factors, its association with localized aggressive periodontitis in young adolescents $^{[24]}$. Dental plaque is the material that adheres to the teeth and consists of bacterial cells (mainly Strept. mutans and Strept. sanguinis), salivary polymers and bacterial extracellular products. Plaque is a biofilm on the surfaces of the teeth. This accumulation of micro-organisms subject the teeth and gingival tissues to high concentrations of bacterial metabolites which results in dental disease. If not taken care of, via brushing or flossing, the plaque can turn into tartar (its hardened form) and lead to gingivitis or periodontal disease. Most of the bacterial species found in the mouth belong to microbial communities, called biofilms, a feature of which is inter-bacterial communication. Cell-cell contact is mediated by specific protein adhesins and often, as in the case of inter-species aggregation, by complementary polysaccharide receptors. Another method of communication involves cell-cell signaling molecules, which are of two classes: those used for intra-spp. and those used for inter-spp. signaling. An example of intra-spp. communication is quorum sensing. Oral bacteria have been shown to produce small peptides, such as competence stimulating peptides, which can help promote 
single-spp. biofilm formation. A common form of inter-spp. signaling is mediated by 4, 5-dihydroxy-2, 3pentanedione (DPD) or Autoinducer-2 (Al-2) ${ }^{[25]}$. Some Lactobacillus spp., have been associated with dental caries although these bacteria are normally symbiotic in humans and are found in the gut flora ${ }^{[26]}$.

The aim of the experiment: The usage of raw Frankincense (Boswellia sacra) at Taif, KSA, with the stress of anti-microbial activities for simple and chip herbal materials which using by all ladies in Arabian region. Taking into our consideration anti-microbial effects, low-cost, availability of Frankincense in the herbal markets, relatively high frequency of use in all ages and making good hygienic condition for oral cavity.

\section{Materials and Methods:}

Frankincense chewing gum preparation: It was chosen and purchased from famous herbal market as a pure type at Taif Souk. It was weighted as separately specimens $(1 \pm 0.2 \mathrm{gm})$ for each understudy persons with extra samples for any dismissing in the experiment.

Understudy persons preparation: Experiment advertise was making at Taif region, it was stay for 2days only. Ten female were accepted as experimental understudy persons, 5 female were selected from them as final chosen for experiment, (healthy, diseased free and don't take any medication). The experiment was explained and consent forms were gotten from them for the experimental work.

Experiment steps: It was started at Zero hr. time considered as control baseline. Specimens were collected from all understudy persons at Zero hr. time for control microbial scan. Then understudy persons started the usage of gum and adjusted the collection of others specimens for everyone hr. till 5 hrs., as well specimens were sent to Microbiology Laboratory for microbial scan.

Microbial scan: Specimens were collected at the time recorded for the experiment. Whole saliva collected by spitting (at least $1 \mathrm{ml}$ ) from each understudy person obtained in sterile container and labeled. Each specimen was seeded onto bacterial and fungal culture media at that moment, or placed immediately into an Eppendorff tube and kept deep-frozen carbon dioxide at $\left(-70^{\circ} \mathrm{C}\right)$ until sent for analysis in Microbial Laboratory by standard methods. Specimens were collected transported to Microbiology Laboratory within $1 \mathrm{hr}$. of collection and processed for microbial scan immediately. Microbial determination for CFUs/ml was performed for understudy persons, CFUs/ml were calculated by: y X $10^{-\mathrm{d}} \mathrm{X} 1 / \mathrm{v}$ (where $\mathrm{d}=$ dilution $\mathrm{v}=$ volume plated and $\mathrm{y}=$ colony count on the plates, between 30 and 300$)^{[27-28]}$.

Data Analysis: The data were recorded and entered into Microsoft excel sheet, then summarized and analyzed $^{[29]}$.

III. Results and discussion:

Table and figure 1: The main effective contents of Frankincense chewing gum

\begin{tabular}{|cc|}
\hline Contents & Main \% \\
\hline Volatile essential oil & $\mathbf{7 \%}$ \\
\hline Water-soluble gum & $\mathbf{2 3 \%}$ \\
\hline Alcohol-soluble resins & $\mathbf{7 8 \%}$ \\
\hline
\end{tabular}

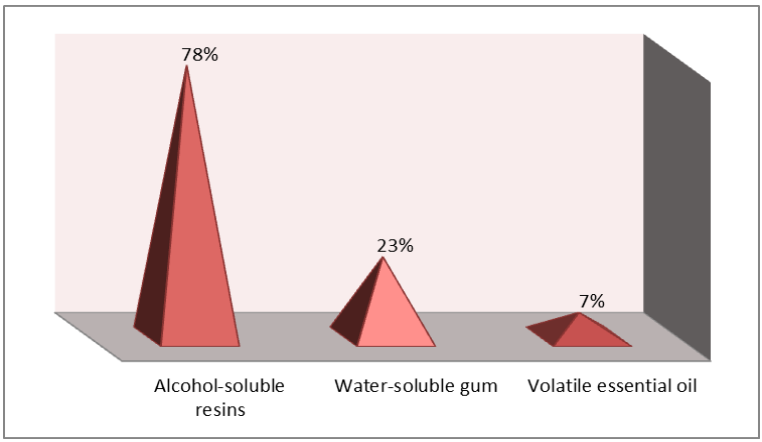

Table and figure 1 show the main effective contents of Frankincense chewing gum were alcoholsoluble resins is $78 \%$, water-soluble gum $23 \%$ and volatile essential oil $7 \%$ respectively. Frankincense (Boswellia sacra) is a tree indigenous to Dhofar region and is one of the most famous plant of Sultanate of Oman. It is also known as Luban, Bakhor or Kendar in Arabic region ${ }^{[1]}$. Its composed of about 5-9\% essential oil, $65-85 \%$ alcohol-soluble resins, and the remaining water soluble gums $30-40 \%{ }^{[2]}$. Frankincense consists of essential oils, gum, and terpenoids. The boswellic acids in the terpenoid portion are the active constituents in Boswellia ${ }^{[10]}$. Boswellic acids are further known to contain $\beta$-boswellic acid, acetyl- $\alpha$-boswellica acid, and acetyl- $\beta$-boswellic acid, and associated 3-acetyl-11-hrdroxy- $\beta$-boswellic acid and 11-keto- $\beta$-boswellic acid 
acetate chemicals $^{[12]}$. AKBA components of the boswellic acids are also identified to be among the biologically active and most potent constituents ${ }^{[13]}$. Boswellic acid and their derivatives were investigated for antiinflammatory. Mono-terpenoids seem to be the dominant class of compounds found in the oils. The main compounds essential oil were $\alpha$-pinene $45.7 \%, \alpha$-terpinene $11.5 \%$ and trans-sabinene hydrate ${ }^{[15]}$. Resin oils showed similar chemical profiles, with iso-incensole and iso-incensole acetate as the main di-terpenic components. Both n-octanol and n-octyl acetate, along with the diterpenic components incensole and incensole acetate, were the characteristic compounds of B. papyrifera oleogum resin oil ${ }^{[16]}$.

Table 2 and figure $(2 \& 3)$ : Microbial contents of the buccal/oral cavity for each person using rankincense chewing gum

\begin{tabular}{|c|c|c|c|c|c|c|c|}
\hline \multirow[t]{2}{*}{ Hr. } & \multicolumn{7}{|c|}{ CFUs/ml } \\
\hline & Person1 & Person2 & Person3 & Person4 & Person5 & The main & Differences \\
\hline $\begin{array}{l}\text { Zero } \\
\text { hr. }\end{array}$ & 13000 & 13200 & 12900 & 12800 & 13100 & $\begin{array}{c}(65000 / 5) \\
13000\end{array}$ & $\begin{array}{l}\text { Zero hr.- } \\
\text { 1hr }\end{array}$ \\
\hline & & & & & & & 4800 \\
\hline $1 \mathrm{hr}$ & 8100 & 8300 & 8200 & 8400 & 8000 & $\begin{array}{c}(41000 / 5) \\
8200\end{array}$ & 1hr.-2hr. \\
\hline & & & & & & & 2100 \\
\hline $2 \mathrm{hr}$. & 6300 & 6100 & 6200 & 5900 & 6000 & $\begin{array}{c}(30500 / 5) \\
6100\end{array}$ & 2hr.-3hr. \\
\hline & & & & & & & 2200 \\
\hline $3 \mathrm{hr}$. & 3900 & 3700 & 4000 & 4100 & 3800 & $\begin{array}{c}(19500 / 5) \\
3900\end{array}$ & 3hr.-4hr. \\
\hline & & & & & & & 1700 \\
\hline 4hr. & 2100 & 2000 & 2400 & 2300 & 2200 & $\begin{array}{c}(11000 / 5) \\
2200\end{array}$ & 4hr.-5hr. \\
\hline & & & & & & & 1460 \\
\hline 5 hr. & 730 & 720 & 750 & 760 & 740 & $(3700 / 5) 740$ & \\
\hline
\end{tabular}
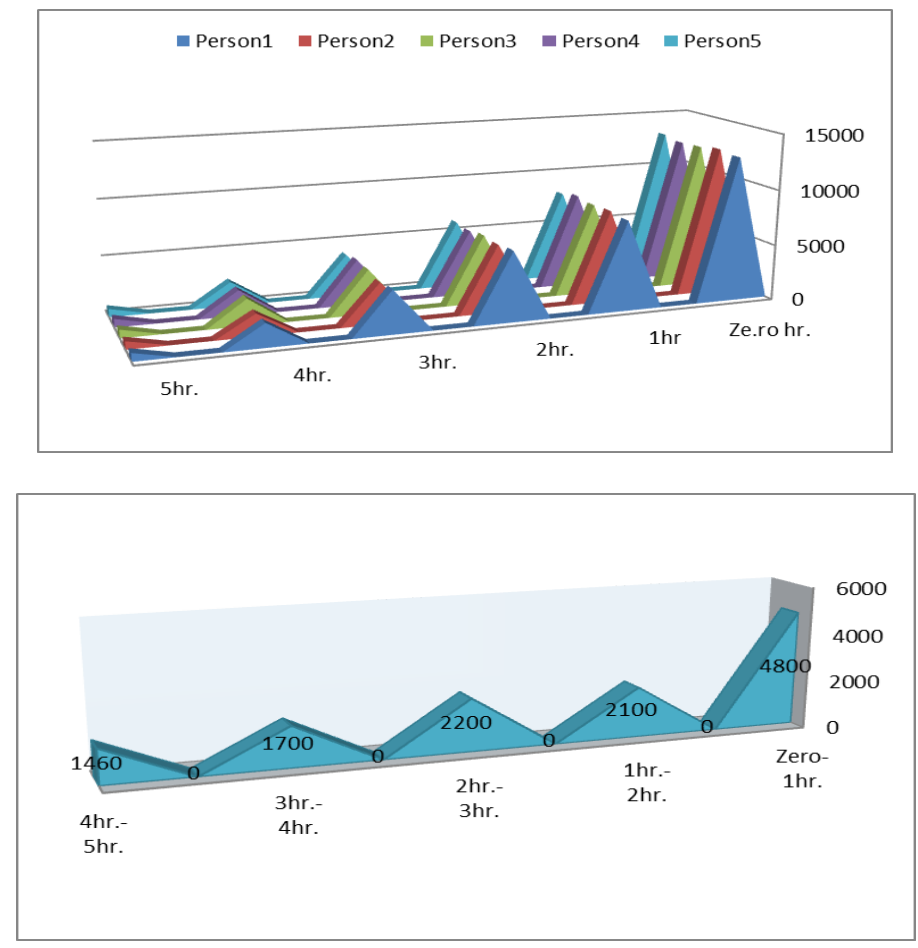

Table 2 and figure $(2 \& 3)$ show microbial contents of the buccal/oral cavity for each person using Frankincense chewing gum. The main CFUs/ml of saliva were 13.000, 8.200, 6.100, 3.900, 2.200, and 740 at Zero, 1, 2, 3, 4, and 5hrs. The differences of CFUs/ml were 4.800, 2.100, 2.200, 1.700, and 1.460 between (Zero hr.-1hr.), (1hr.2hr.), (2hr.-3hr.), (3hr.-4hr.), and (4hr.-5hr.) respectively. 
Table 3 and figure 4: Incidence of the main microbial contents of the buccal/oral cavity with using Frankincense chewing gum

\begin{tabular}{|c|c|c|}
\hline Hrs. & $\begin{array}{l}\text { The main } \\
\text { CFUs/ml }\end{array}$ & $\begin{array}{c}\text { The main } \\
\text { No./ml }\end{array}$ \\
\hline Zero hr. & $1.3 \times 10^{4}$ & 13.000 \\
\hline 1hr. & $8.2 \times 10^{3}$ & 8.200 \\
\hline $2 \mathrm{hr}$. & $6.1 \times 10^{3}$ & 6.100 \\
\hline $3 \mathrm{hr}$. & $3.9 \times 10^{3}$ & 3.900 \\
\hline 4hr. & $2.2 \times 10^{3}$ & 2.200 \\
\hline $5 \mathrm{hr}$. & $7.4 \times 10^{2}$ & 740 \\
\hline
\end{tabular}

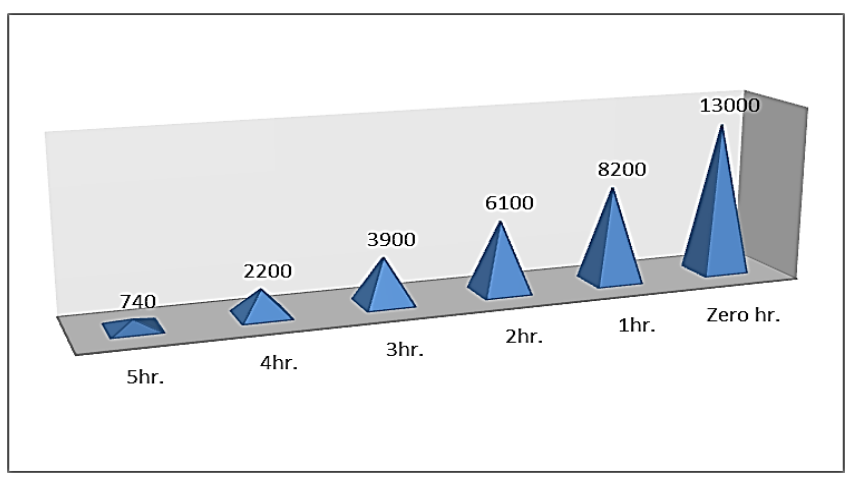

Table 3 and figure 4 show incidence of the main microbial contents of the buccal/oral cavity with using Frankincense chewing gum. CFUs $/ \mathrm{ml}$ were $1.3 \mathrm{X} 10^{4}, 8.2 \mathrm{X} 10^{3}, 6.1 \mathrm{X} 10^{3}, 3.9 \mathrm{X} 10^{3}, 2.2 \mathrm{X} 10^{3}$, and $7.4 \mathrm{X} 10^{2}$ at Zero, $1,2,3,4,5 \mathrm{hr}$. respectively. Non-steroidal anti-inflammatory drugs (NSAIDS) used for inflammatory conditions, among the constituents of boswellic acid, $\beta$-boswellic acids (the major constituents of boswellic acids) was shown to have anti-inflammatory and anti-arthritic pain activity, which was found to be due to their ability to inhibit 5-lipoxygenase activity ${ }^{[9]}$, also identified to be among the biologically active and most potent constituents $^{[13]}$. Boswellic acid and their derivatives were investigated for anti-inflammatory. Essential oil showed anti-bacterial, anti-fungal and immuno-stimulating activity ${ }^{[14]}$. The anti-microbial activities of the essential oils were individually evaluated against fungi, Gram-positive and Gram-negative bacteria strains. Resins destroy microbial cell wall and stop protein synthesis in Strept. salivaris, Staph. aureus, and Bacillus megaterium $^{[16]}$. Pharmacological activities of Frankincense, as crude extracts, the distilled essential oil and isolated compounds reported to exhibit in-vitro anti-bacterial, anti-fungal and immuno-modulatory activity. Volatile oils make sensitization of microbial cell contents and have anti-inflammatory effect on Staph. aureus, Staph. epidermidis, Staph. hominis, Bacillus cereus, E. coli, Proteus vulgaris and Candida albicans ${ }^{[17]}$. Frankincense application can lead to remarkable decrease in inflammatory indices in comparison to drug therapy. It's a safe and low cost herbal medicine, may be feasibly applied to improve inflammation based disease of gingival as an adjunct to the conventional mechanical therapy ${ }^{[18]}$. The human mouth is an ideal environment of the existence and growth of micro-organisms. It provides a source of water and nutrients, as well as a moderate temperature. Resident bacteria of the mouth adhere to the teeth and gums to resist mechanical flushing from the mouth to stomach where they are destroyed by hydrochloric acid ${ }^{[21]}$. Anaerobic bacteria in the oral cavity include: Actinomyces, Bacteriodes, Bifidobacterium, Eubacterium, Fusobacterium, Lactobacillus ${ }^{[22]}$. The role of oral micro-organisms in the two major dental diseases: dental caries and periodontal disease ${ }^{\text {[23] }}$.

\section{Conclusion}

Frankincense has been indicated to have wide pharmacological and industrial potentials. Result of this experiment displayed anti-microbial properties on microbial contents of buccal/oral cavity as a source of infection. This herbal product may be feasibly and safely applied to improve mouth hygiene for all ages.

\section{Acknowledgement}

Our thankful send to understudy female persons for helping in the experiment, also to laboratory staff for their work. 


\section{References:}

[1]. Wallis, T., 1967. Text Book of Pharmacognosy, $5^{\text {th }}$ Edition, J. \& A. Churchill Ltd., London, PP: 500-501.

[2]. Murthy, T. and Shiva, M., 1977. Salai guggul from Boswellia serrata Roxb-Its exploitation and utilization. Indian Forester, 103: 466-474.

[3]. Wahab, A., Aboutabl, E., El-Zalabani, S., Fouad, H., De Pooter, H. and El-Fallaha, B., 1987. The essential oil of Olibanum. Plant. Med., 53: 382-384.

[4]. Vollesen, K., 1989. Burseraceae. In Flora of Ethiopia. Vol. 3. Hedberg, I. and Edwards, S. (eds.) Addis Ababa and Asmara, Ethiopia, PP: 442- 478

[5]. Martinez, D., Lohs, K., Janzen, J., Weihrauch, A and Bedeutung, B., 1989. Medizinr Stuttgart: Wissenschaftliche Verlagsgestellschaft.

[6]. Chiavari, G., Galletti, G., Piccagali, R. and Mohammed, M., 1991. Differential between resins of Boswellia carteri and Boswellia frereana (Frankincense of omali origin. J. Ess. Oil Res., 3: 185-186.

[7]. Safayhi, H., Mack, T. and Saieraj, J., 1992. Boswellic acids: Novel, specific, nonredox inhibitors of 5-lipoxygenase. J. Pharmacol. Exp. Ther., 261: 1143-1146.

[8]. Ammon, H., Safayhi, T., Mark, T. and Sabieraj, J.,1993. Mechanism of anti-inflammatory actions of curcumine and boswellic acids. J. Ethnopharamacol., 38: 113-119.

[9]. Majeed, M., Badmaev, V., Gopinathan, S., Rajendran, R. and Norton, T., 1996. Boswellin, the Anti-inflammatory Phytonutrient. Nutriscience Publishers, Inc., Piscataway, NJ.

[10]. Safayhi, H., Sailer, E. and Amnon, H., 1996. 5-lipoxygenase inhibition by acetyl-11-keto-bboswellic acid. Phytomed., 3: 71-72.

[11]. Schauss, A., Milholland, R. and Munson, S., 1999. Indian Frankincense (Boswellia serrata) gum resin extract: a review of therapeutic applications and toxicology, Nat. Med. J., 2 :16-20.

[12]. Huang, M., Badmaev, V., Ding, Y., Liu, Y., Xie, J. and Ho, C-T., 2000. Anti-tumor and anti-carcinogenic activities of triterpenoid, $\beta$-boswellic acid. Biofactors, 13: 225-230.

[13]. Krieglstein, F., Anthoni, K., Rijcken, E., Laukötter, M., Spiegel, H. and Boden, S., 2001. Acetyl-11-keto- $\beta$-boswellic acid, a constituent of a herbal medicine from Boswellia serrata resin, attenuates experimental ileitis. Nant glioma: induction of apoptosis but no modulation of drug sensitivity. Br. J. Cancer, 80: 756-765.

[14]. Ota, M., and Houghton, P., 2005. Boswellic acid with acetylcholinesterase inhibitory properties from frankincense" $53^{\text {rd }}$ annual congress organized by society of medicinal plants. Societa Italiana di Fitochimica Florence, PP: 339.

[15]. Kubmarawa, D., Ogunwande, I., Okorie, D., Olawore, N. and Kasali, A., 2006. Constituents of the essential oils of Boswellia dalzielii Hutch. Nigeria. J. Ess. Oil Res., 18: 119-120.

[16]. Lorenzo Camarda, T., Vita, D., Rosa, P. and Domenico, S., 2007. Chemical Composition and Antimicrobial Activity of Some Oleogum Resin Essential Oils from Boswellia SPP. (Burseraceae). Annali di Chimica., 97: 837-844.

[17]. Ali, A., Wurster, M., Arnold, N., Teichert, A. and Schmidt, J., 2008. Chemical Composition and Biological Activities of Essential Oils from the Oleogum Resins of Three Endemic Soqotraen Boswellia Species. Rec. Nat. Prod., 2: 6-12.

[18]. Khosravi, S., Mahmoodian, H. and Moghadamnia, A., 2011. The effect of Frankincense in the treatment of moderate plaqueinduced gingivitis: a double blinded randomized clinical trial. DARU., 19: 288-294

[19]. Fatouma, M., Louis, O., Imael H. and Mamoudou, H., 2012. Antimicrobial activities of essential oil and methanol extract of Boswellia sacra Flueck. and Boswellia papyrifera (Del.) Hochst from Djibouti. Int. J. Management, Modern Sci. and Tec., 1: 1-10.

[20]. Salwa, A. and Shah. A., 2013. Evaluation of Knowledge and Practices of Omani population in Sur region about the beneficial uses of Frankincense - a commonly used natural plant product. Scholars Academic Journal of Pharmacy (SAJP),Sch. Acad. J. Pharm., 2: $168-172$

[21]. Willey, J., Sherwood, L., Woolverton, C., 2011. Prescott's Microbiology (8 ${ }^{\text {th }}$ ed.). New York: McGraw Hill, PP: 731-737

[22]. Sutter, V., 1984. Anaerobes as normal oral flora. Reviews of infectious diseases, 6: S62-S66.

[23]. Rogers, A., 2008. Molecular Oral Microbiology. Caister Academic Press.

[24]. Fine, D., 2008. Molecular Windows into the Pathogenic Properties of Aggregatibacter Actinomycete mcomitans. Molecular Oral Microbiology. Caister Academic Press.

[25]. Rickard, A., 2008. Cell-cell Communication in Oral Microbial Communities. Molecular Oral Microbiology. Caister Academic Press.

[26]. Ljungh A. and Wadstrom, T., 2009. Lactobacillus Molecular Biology: From Genomics to Probiotics. Caister Academic Press.

[27]. World Health Organization., 2004. WHO Laboratory Biosafety Manual. 3rd ed. Geneva: World Health Organization.

[28]. Cai, Z., C. Niladri, J. Wenchao, C. Conrad, P. Jean-Philippe and M. Raymond, 2011. Optimized digital counting colonies of clonogenic assays using Image software and customized macros: comparison with manual counting. Int. J. of Radiation Bio., 87: 1135-1146.

[29]. Coulombier, D., Fagan, R., Hathcock, L. and Smith, C., 2001. Epi Info 6 Version 6.04.A Word Processing, Database and Statistical Program for Public Health. Centers for Disease Control and Prevention, Atlanta, Delaware, USA. 